\title{
Hypervigilance for fear after basolateral amygdala damage in humans
}

\author{
D Terburg ${ }^{1,2}$, BE Morgan ${ }^{3}$, ER Montoya ${ }^{1}$, IT Hooge ${ }^{1}$, HB Thornton ${ }^{2}$, AR Hariri ${ }^{4}$, J Panksepp $^{5}$, DJ Stein ${ }^{2}$ and J van Honk ${ }^{1,2}$
}

Recent rodent research has shown that the basolateral amygdala (BLA) inhibits unconditioned, or innate, fear. It is, however, unknown whether the BLA acts in similar ways in humans. In a group of five subjects with a rare genetic syndrome, that is, Urbach-Wiethe disease (UWD), we used a combination of structural and functional neuroimaging, and established focal, bilateral BLA damage, while other amygdala sub-regions are functionally intact. We tested the translational hypothesis that these BLAdamaged UWD-subjects are hypervigilant to facial expressions of fear, which are prototypical innate threat cues in humans. Our data indeed repeatedly confirm fear hypervigilance in these UWD subjects. They show hypervigilant responses to unconsciously presented fearful faces in a modified Stroop task. They attend longer to the eyes of dynamically displayed fearful faces in an eyetracked emotion recognition task, and in that task recognize facial fear significantly better than control subjects. These findings provide the first direct evidence in humans in support of an inhibitory function of the BLA on the brain's threat vigilance system, which has important implications for the understanding of the amygdala's role in the disorders of fear and anxiety.

Translational Psychiatry (2012) 2, e115; doi:10.1038/tp.2012.46; published online 15 May 2012

\section{Introduction}

The human amygdala is critically involved in social and emotional behavior, and has a vital role in the assessment of, and responding to, threat. ${ }^{1}$ The amygdala is, however, not a homogeneous brain region, but consists of several sub-nuclei that are so different in structure and connectivity that they are best regarded separately. ${ }^{2-4}$ Direct evidence into the role of amygdala sub-regions in threat processing comes, however, predominantly from rodent lesion research. ${ }^{5,6}$ Although human cases of damage to the basolateral amygdala (BLA), ${ }^{7,8}$ and more extended or complete amygdala damage have been described, ${ }^{9-15}$ there is to our knowledge no causal evidence pertaining to amygdala sub-region function in human fear processing.

The human amygdala is thought to promote fast and efficient responding to threat within a brainstem-amygdalacortical alarm system (for example, Liddell et al. ${ }^{16}$ ), and rodent studies suggest that the BLA has been especially implicated in such threat processing. In rodents the BLA is essential for the acquisition and extinction of conditioned fear, ${ }^{6,17,18}$ but there is increasing evidence that the rodent BLA also inhibits unconditioned and acute fear responses. ${ }^{19-23}$ Unconditioned fear reveals itself as acute fear or panic in humans, ${ }^{4,24}$ and the PAG (periaqueductal gray) is critically involved in the instigation of such fear and panic responses. ${ }^{4}$ Humanneuroimaging data show that both the PAG and the centralmedial amygdala (CMA) are activated when threats are imminent and unavoidable. When a threat can be avoided, however, activation shifts to the BLA and prefrontal cortex
(PFC), which is thought to underlie threat estimation and response inhibition. ${ }^{25-27}$ It could be argued that the inhibitory role of the BLA on responsiveness to innate threat cues in rodents ${ }^{19-23}$ is responsible for this switch in activity from acute fear responding in the midbrain to cortical threat estimation, but it is unknown whether this evidence from rodents can be translated to humans. Nonetheless, it has been shown numerous times that the amygdala indeed responds to facial expressions of fear, ${ }^{28}$ which are the prototypical innate threat cues for humans, whereby BLA activity has been specifically linked to unconscious processing of facial fear. ${ }^{29}$ Research addressing the question whether in congruence with rodent research the human BLA inhibits the fear response to such innate threat cues, is, however, lacking.

In the present study, we tested a group of five women with Urbach-Wiethe disease (UWD). UWD is a rare geneticdevelopmental disorder characterized by focal calcifications in the bilateral amygdalae, which provides a unique window onto human amygdala function..$^{30}$ Early evidence from a UWD subject with full amygdala damage suggested a specific role for the amygdala in the recognition of static displays of facial fear, ${ }^{9,10}$ which later was shown to stem from an inability to automatically maneuver visual attention from the mouth to the emotionally critical eye region of static faces. ${ }^{31}$ Indeed, neuroimaging data show that the amygdala is active when gaze is shifted from mouth to eyes, ${ }^{32}$ and is triggered specifically by fearful eyes, ${ }^{33}$ but fear processing findings in UWD have been inconsistent, ${ }^{15,34}$ possibly reflecting heterogeneity in size, location and epileptogenicity of the amygdala lesions. $^{7}$

\footnotetext{
1'Department of Psychology, Utrecht University, Utrecht, The Netherlands; ' 2 Department of Psychiatry and Mental Health, University of Cape Town, Cape Town, South Africa; ${ }^{3}$ MRC Medical Imaging Research Unit, Department of Human Biology, University of Cape Town, Cape town, South Africa; ${ }^{4}$ Department of Psychology and Neuroscience, Institute for Genome Sciences and Policy, Duke University, Durham, NC, USA and ${ }^{5}$ Department of Psychology, Washington State University, Pullman, WA, USA

Correspondence: D Terburg, Department of Psychology, Utrecht University, Heidelberglaan 2, Utrecht 3584CS, The Netherlands.

E-mail: d.terburg@uu.nl

Keywords: basolateral amygdala; eye tracking; fear vigilance; fMRI; sMRI; urbach-wiethe disease

Received 2 March 2012; revised 16 April 2012; accepted 16 April 2012
} 
First, we will show that the selective bilateral calcifications in the brains of our five UWD subjects are limited to the BLA. We used high-resolution structural MRI to assess the relative location and extent of calcified damage, and functional MRI to assess the reactivity of the intact amygdala sub-regions. Next, we tested the crucial hypothesis that these UWD subjects are hypervigilant for subliminal fear, which would support the hypothesis that the human BLA has a role in the inhibition of acute responding to innate threat cues. UWD subjects and a carefully matched group of healthy volunteers performed in a modified emotional Stroop paradigm that directly taps into threat-driven attentional processing, ${ }^{35}$ and can validly assess threat hypervigilance by using subliminally presented fearful faces as stimuli. ${ }^{36,37}$ Finally, we assessed the ability of UWD subjects and controls in emotion recognition of ecologically valid dynamic expressions and measured their eye movements to assess mechanisms of visual attention.

\section{Materials and methods}

Ethics statement. This study was approved by the Health Sciences Faculty Human Research Ethics Committee of the University of Cape Town. All participants provided written informed consent.

Participants. We tested five women, without any (history of) secondary psychopathology or epileptic insults, from a previously described UWD cohort in South Africa, ${ }^{34}$ where this genetic disorder is most prevalent. ${ }^{15}$ UWD subjects were compared against a group of healthy volunteers $(N=16)$ matched for gender, age and IQ, and living in the same area of South Africa, that is, mountain-desert villages near the Namibian border. Twelve of these participants took part in the subliminal fear-vigilance task and the dynamic emotion recognition task. Eight of them, and an additional four healthy volunteers, took part in the static emotion-rating task that was conducted $\sim 2$ years later. Demographic data are summarized in the Supplementary Information (Supplementary Information, Supplementary Tables S1 and S2), as well as details and issues regarding IQ testing in this nonWestern sample. Statistics are two-tailed non-parametric Mann-Whitney $U$-tests with $\alpha=0.05$, and effect size $(r)$ for significant effects, throughout the behavioral data analysis.

Structural MRI assessment. MRI scans were acquired with a Siemens Magnetom Allegra 3-Tesla head-only scanner (Siemens Medical Systems GmBH, Erlangen, Germany) at the Cape Universities Brain Imaging Centre (CUBIC) in Cape Town, South Africa. Structural whole brain T2-weighted MRI scans were obtained with $1 \mathrm{~mm}$ isotropic resolution, $\mathrm{TR}=$ $3500 \mathrm{~ms}$ and TE $=354 \mathrm{~ms}$. T2-weighted scans of all five UWD subjects were normalized to MNI space using the unified model as implemented in SPM5 (http://www.fil.ion.ucl.ac.uk/spm), which is optimized for normalization of lesioned brains. ${ }^{38}$ Subsequently the extent of the calcifications was determined with the 3D volume-of-interest feature implemented in MRIcroN (http://www.cabiatl.com/mricro/mricron).

On the basis of MR images the precise borders between amygdalae and neighboring structures, or between the sub-regions of the amygdala, cannot be established. ${ }^{39,40}$ To determine the precise location of the lesions in our UWD subjects, we therefore assigned the lesion volumes to cytoarchitectonic probability maps according to the method described by Eickhoff et al. ${ }^{41}$ In this method, that is implemented in the SPM5 anatomy toolbox (http://www. fz-juelich.de/inm/inm-1/spm_anatomy_toolbox), a volume of interest (VOI) is superimposed onto a cytoarchitectonic probability map of the amygdala and hippocampus. ${ }^{39}$ This map is based on microscopic analyses of 10 postmortem human brains and follows a generally accepted division of the human amygdala in three sub-regions. The first is the CMA, which consists of the central and medial nuclei. The second is the BLA, which includes the lateral, basolateral, basomedial and paralaminar nuclei and the third is the superficial (or corticoid) amygdala (SFA), which includes the anterior amygdaloid area, amygdalopyrifom transition area, amygdaloid-hippocampal area and the cortical nucleus. ${ }^{39}$ This method assigns to any given voxel a value representing the probability that it belongs to an underlying structure. These are derived from an overlap analysis of 10 postmortem brains, and are therefore divided in 10 separate probability classes ranging from 10 to $100 \%$ probability. For each probability class of each structure that shares voxels with the VOI, the 'observed versus expected' class representation is computed. This value represents how much more (or less) that class is observed in the VOI compared with what could be expected from the entire probability map of that structure, and is computed with the following equation:

$$
P_{o-e}=\frac{P_{o}-P_{e}}{P_{e}}
$$

whereby $P_{\mathrm{o}-\mathrm{e}}$ represents the 'observed versus expected' class representation, $P_{\mathrm{o}}$ represents the percentage of VOI voxels in that class, and $P_{\mathrm{e}}$ represents the percentage of voxels from that class in the whole cytoarchitectonic map of that structure. The outcome values thus indicate which class is overrepresented in the VOI relative to the whole cytoarchitectonic map.

To estimate how well the lesion volumes fit to the underlying structure, $P_{\text {excess }}$ values are computed using the following equation:

$$
P_{\text {excess }}=\frac{P_{s(\text { vol })}}{P_{s(t o t a l)}}
$$

whereby $P_{\mathrm{s}(\mathrm{VO})}$ represents the average cytoarchitectonic probability of the voxels that are shared by the structure and the VOI, and $P_{\mathrm{s} \text { (total) }}$ represents the average probability of the whole structure's cytoarchitectonic map. These values thus represent how much the average probability of the overlapping voxels exceed the overall probability distribution of that structure, and thus indicate whether the VOI overlaps with relatively high or low probability classes of that structure. In other words, $P_{\text {excess }}$ represents how 'central' the location of the VOI is relative to that structure's cytoarchitectonic map, whereby $P_{\text {excess }}>1$ indicates a more central, and $P_{\text {excess }}<1$ a more peripheral location. ${ }^{41}$ 
Functional MRI assessment. Functional whole brain MRI scans were obtained with a 2D-EPI sequence with 36 slices in interleaved-ascending order, $3.5 \mathrm{~mm}$ isotropic resolution, flip-angle $=70^{\circ}, \mathrm{TR}=2000 \mathrm{~ms}, \mathrm{TE}=27 \mathrm{~ms}$ and $E P I-f a c t o r=64$. The first four volumes were acquired before the start of the fMRI task, and discarded from analyses.

Participants viewed a trio of faces and matched emotional expressions by choosing one of the two lower pictures (either an angry or a fearful face) that expressed the same emotion as the picture on top. This condition was interleaved with a sensori-motor control condition involving the matching of oval shapes. ${ }^{42}$ To increase cultural validity, gray-scaled face-stimuli included Caucasian as well as African-American actors, ${ }^{43}$ and the shape-stimuli were constructed from scrambled face stimuli to match visual contrast levels.

The task was presented in a blocked design, with five shape-matching, interleaved with four emotion-matching, blocks, with six $5 \mathrm{~s}$ trials each and always including faces of one gender only. All face stimuli were presented equally often as target, match or non-match in randomized order. Each block was preceded by the instruction 'match emotion' or 'match shape' (in Afrikaans) for $2 \mathrm{~s}$, making a total task duration of $288 \mathrm{~s}$. Participants responded by a button press with either the left or right hand, corresponding to the position of the match stimulus.

Analyses were performed with SPM5 (http://www.fil.ion.ucl. ac.uk/spm). For each participant all volumes were realigned to the first volume, and co-registered to the structural T2weighted volume. ${ }^{44}$ Subsequently, the resulting functional images were normalized to MNI-space using the parameters obtained from the structural analysis, and smoothed with a full-width-half-maximum Gaussian kernel of $8 \times 8 \times 8 \mathrm{~mm}$. Contrast maps for match-emotion $>$ match shape were obtained with realignment parameters and high-pass filter (cutoff 128s) entered as regressors of no interest. For group level statistics these were entered in a one-sample $t$-test analysis. Functional activation of the amygdala was assessed bilaterally within regions of interest (ROIs) of the BLA and the combined CMA and SFA. ROIs were constructed based on the cytoarchitectonic probability maps as implemented in the anatomy toolbox for SPM5. ${ }^{39,45}$ We applied an extent threshold of 10 voxels, and significance threshold was set at $P<0.05$ (false-discovery rate corrected). This rather lenient threshold is justifiable given that we presently only assess whether the amygdala's sub-regions are responsive in general.

Behavioral assessment: subliminal fear vigilance task. Participants verbally named as quickly as possible the color of backwardly masked fearful, happy and neutral faces, ${ }^{36,37}$ whereby a generic slow down in color naming of threatrelated information is reliably associated with automatic vigilance to threat. $^{35}$ After a fixation cross $(750 \mathrm{~ms})$, randomly 1 of 90 face stimuli ${ }^{46}$ ( 5 male and 5 female, 3 emotions, colored in red, green or blue) was presented for $14 \mathrm{~ms}$, before being replaced by a masking stimulus. Intertrial interval was $1500-2500 \mathrm{~ms}$. Masking stimuli were randomly cut, reassembled and rephotographed pictures of the faces. Color naming latencies $>2$ s.d. from the individual means were removed $(4.6 \%)$.
Afterwards, participants performed on an objective awareness check. This was a three-alternative forced-choice emotion recognition task, using the same masked stimuli from the original task, which establishes awareness of the measure of interest, emotional expression. ${ }^{47,48}$

Behavioral assessment: dynamic emotion task. Participants were presented with clips of faces, two male and two female actors, ${ }^{46,49}$ morphing fluidly from neutral to emotional (anger, disgust, fear, happiness, sadness and surprise), and were instructed to choose which of six emotional adjectives (Afrikaans translation of angry, disgusted, fearful, happy, sad and surprised) best described each face. The final frame remained visible until the participant responded with a button press. Emotional intensity of the final image in the sequence ranged from 20 to $100 \%$, in steps of $10 \%$ in consecutive blocks, with all stimuli randomized within each block. Accordingly the duration of the video clips ranged from $0.3 \mathrm{~s}$ in the first to $1.7 \mathrm{~s}$ in the final block with full-blown emotions. The clips were presented within a visual angle of $10^{\circ}$ and eye movements were recorded from clip start until the participant's response with a Tobii-1750 binocular infrared eye tracker (Tobii Technology, Danderyd, Sweden; $50 \mathrm{~Hz}, 0.5^{\circ}$ accuracy).

Performance data on the full-blown (100\%-morphed) trials served as a measure of emotion recognition accuracy. Additionally, for each actor and emotion the morphing percentage after which the emotion is consistently recognized was determined. These were averaged to obtain individual sensitivity scores for each emotion.

Gaze fixations were defined as the average location of all subsequent gaze points within $2^{\circ}$ visual angle and with $a$ minimal duration of $60 \mathrm{~ms} .{ }^{50}$ Fixations within oval areas drawn around the mouth and both eyes separately of the stimuli were used to compute average fixation duration and proportion fixations to these areas relative to all fixations on the face.

Behavioral assessment: static emotion task. To facilitate comparison with earlier studies on UWD, our subjects performed in a similar emotion-rating task as reported in earlier studies. ${ }^{9,10,31}$ A full account of the design of this task and the results are reported in the Supplementary Information.

\section{Results}

Structural MRI assessment. As depicted in Figure 1, amygdala calcification appears to progress with age. ${ }^{51}$ Calcified brain tissue is localized in the BLA (see Figure 2), whereby the lesions in the two oldest subjects possibly extend into the borders of the right SFA. Crucially, in all subjects the CMA seems unaffected by the calcifications.

In a quantitative analysis these results are confirmed. Figure 3 shows $P_{\mathrm{o}-\mathrm{e}}$ and $P_{\text {excess }}$ values for the individual lesions, and for the cluster of voxels where all lesions overlap. From Figure 3 we can make three observations. (1) The structures that might be affected by the lesion. (2) Which probability classes of those structures are most, or least, affected. (3) How 'central' the lesions are to the probability distributions of the underlying structures, represented by 
$P_{\text {excess }}$ values. For the lesioned tissue in the UWD subjects $P_{\text {excess }}$ reached values of $2.17,2.33,2.31,2.13$ and 2.08 in the left BLA, and 1.48, 2.05, 1.93, 1.58 and 1.51 in the right, as a function of chronological age. For the lesion-overlap volumes $P_{\text {excess }}$ reached values of 2.38 and 2.24 for the left and right

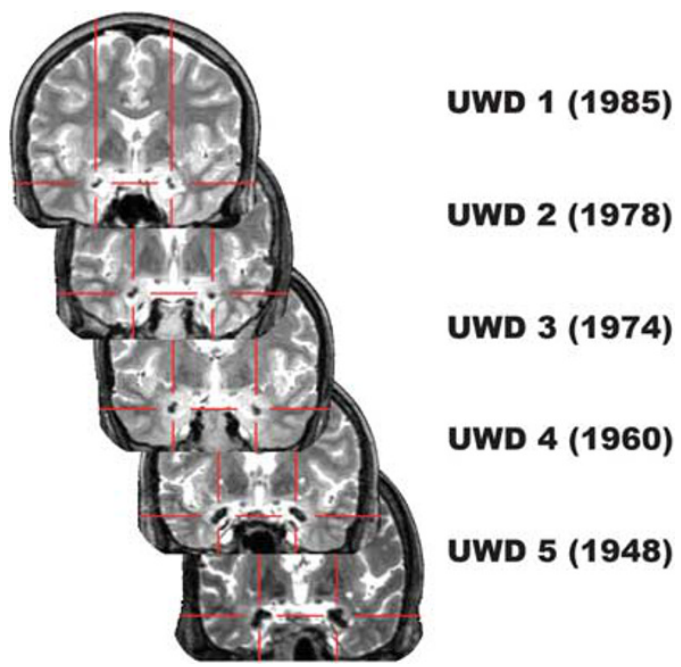

Figure 1 T2-weighted magnetic resonance (MR) images (coronal view) of the five Urbach-Wiethe disease (UWD) subjects with birth year and crosshairs indicating the calcified brain damage.
BLA, respectively, while $P_{\text {excess }}$ values for all other structures was $<0.6$. Thus, as can also be seen in Figure 3 , the lesions are, bilaterally, most central to the BLA, whereby the left-sided lesions are centered in the area with $100 \%$ BLA probability, and the right-sided lesions in the area with $90 \%$ BLA probability. Moreover, for all lesion volumes $P_{\text {excess }}$ values were highest for the BLA (all exceeding 2.0 for the left-sided and 1.5 for the right-sided lesions).

As this method is purely based on probability distributions, it is impossible to fully exclude that other structures than the $B L A$ are affected by the calcifications. The fact that the lesion volumes largely overlap with high probability classes in the bilateral BLA, and that $P_{\text {excess }}$ values greatly exceed the value of 1 , can, however, be seen as strong support for our claim that these UWD subjects have bilateral damage limited to the BLA. We must, however, note that we cannot fully exclude that in the two oldest subjects the calcifications might extend into neighboring structures. Namely, in subject UWD 4 the right SFA ( $\left.P_{\text {excess }}=1.07\right)$, and in subject UWD 5 the left hippocampus (Cornu Ammonis: $P_{\text {excess }}=1.01$ ), left Hippocampal-Amygdaloid Transition Area $\left(P_{\text {excess }}=1.06\right)$, right SFA $\left(P_{\text {excess }}=1.19\right)$ and right Entorhinal Cortex $\left(P_{\text {excess }}=1.19\right)$. Based on $P_{\text {excess }}$ for the CMA (all $\left.<0.5\right)$ we can, however, safely conclude that this structure is relatively unaffected by the bilateral calcifications found in these UWD subjects. Finally, visual inspection of the T2-weighted scans (MRIcroN, http://www.cabiatl.com/mricro/mricron) revealed likely age-related atrophy in the putamen of subject UWD 4.

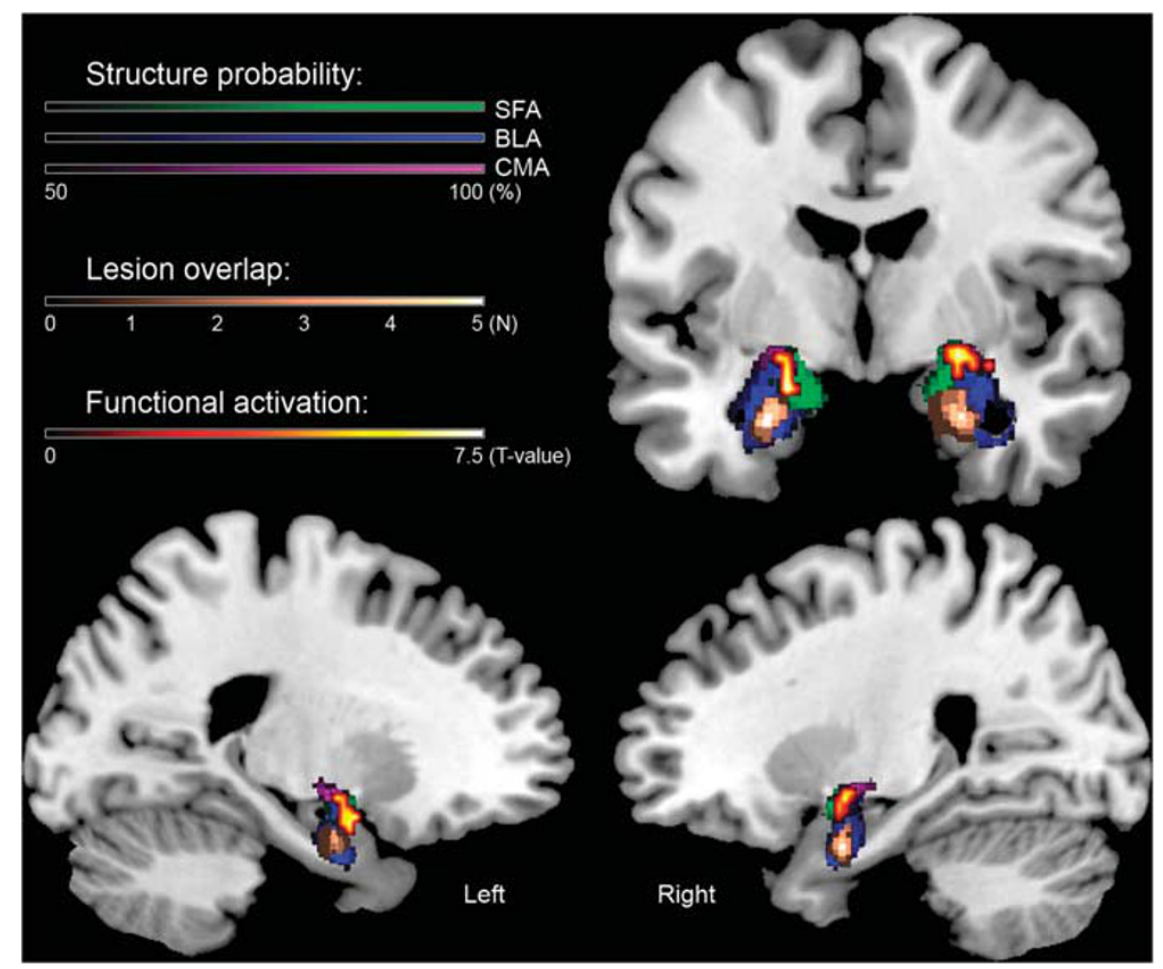

Figure 2 Structural and functional magnetic resonance imaging (MRI) assessment of the bilateral amygdala in our group of five subjects with Urbach-Wiethe disease (UWD). Plotted are the cytoarchitectonic probability-maps of the amygdala thresholded at $50 \%,{ }^{39}$ structural lesion overlap and functional activation during the emotionmatching task (contrast: match emotion $>$ match shape, $P<0.05$, false-discovery rate (FDR) corrected) on a template brain. The structural method indicates that the lesions of the five UWD-subjects are located in the basolateral amygdala (BLA), whereas the functional method shows activation during emotion matching in the superficial amygdala (SFA) and the central-medial amygdala (CMA), but not in the BLA. 
Hemisphere: Left:

Structure: Amy: Hip:
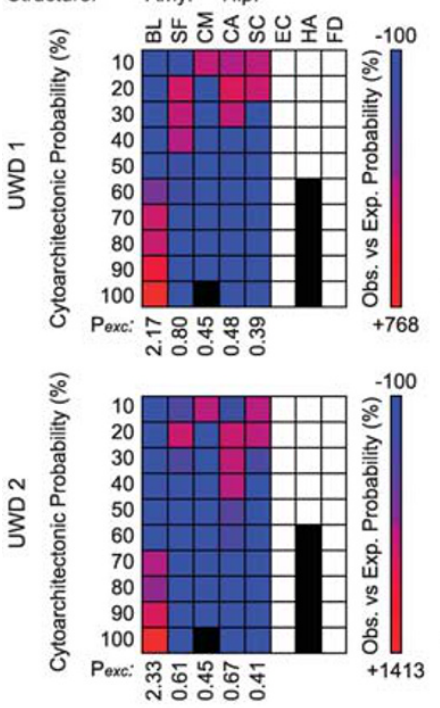

$-100$
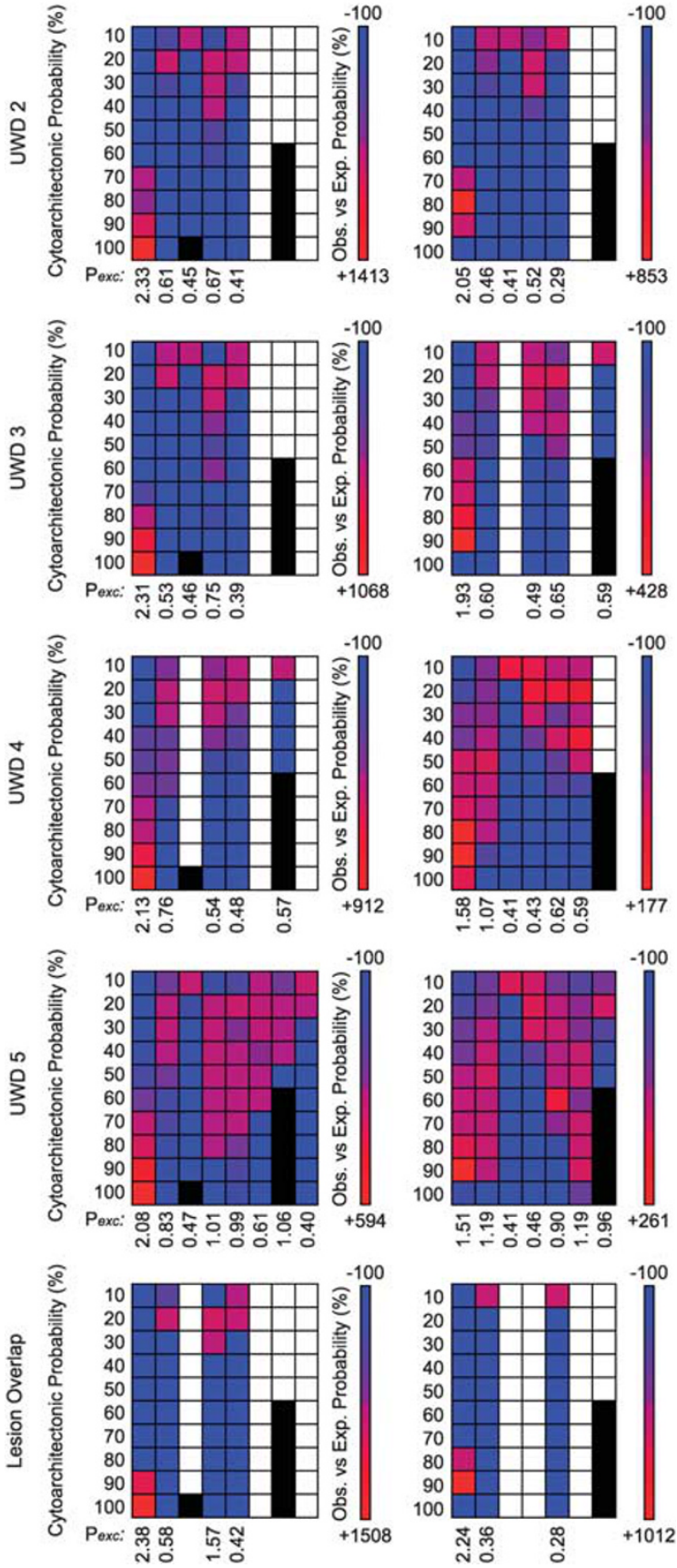

The putamen is, however, not directly involved in processing of fearful expressions, ${ }^{52}$ and therefore not of immediate relevance to the present study.

Functional MRI assessment. ROI analysis $(P<0.05$, falsediscovery rate corrected, see Figure 2) revealed no significant activation clusters in the bilateral BLA, but significant activation of 40 (left), and 35 (right) voxels in ROIs consisting of the amygdala excluding the BLA. Thus, although the BLA is damaged in these subjects, the remaining amygdala tissue seems to be functional.

Behavioral assessment: subliminal fear vigilance paradigm. Color-naming latencies on fear trials were referenced against the other emotions, to create either positive or negative attentional bias scores representing threat vigilance or avoidance, respectively. ${ }^{36,37}$ Overall, UWD subjects and controls were equally fast in color naming ( $540 \mathrm{~ms}$ vs $524 \mathrm{~ms}, U=21, P=0.383$ ). Crucially, fear-bias scores were significantly higher for the UWDsubjects group (fear-neutral; $U=8, P=0.020, r=0.56$, fearhappy; $U=5, P=0.008, r=0.64$, see Figure 4a), indicating that color naming in the UWD group was significantly slowed down when, subliminally, a fearful face was presented. Subsequently, we applied a strict neutral baseline correction by computing separate fear-neutral bias-scores for trials that were preceded by neutral trials, which eliminates trialby-trial emotional conflict. ${ }^{53,54}$ On this pure measure of fear hypervigilance the UWD subjects again showed significant fear-interference $(U=5, P=0.008, r=0.64$, see Figure 4a).

None of the participants reported awareness of the facial expressions, but one control subject scored above chance level on the awareness check (15 correct answers on 30 3-alternative trials, one-tailed binomial-test; $P=0.040$ ). For the remaining participants emotion awareness-check performance was not different from chance-level (10.5 vs 10.6 correct answers), and all group differences on fear hypervigilance remained significant after excluding this participant (fear-neutral; $U=8, P=0.027, r=0.55$, fear-happy; $U=5$, $P=0.011, r=0.64$, fear-neutral baseline corrected; $U=5$, $P=0.011, r=0.64)$.

Behavioral assessment: dynamic emotion task. Table 1 provides performance scores (accuracy and sensitivity) on

Figure 3 Observed versus expected probability matrices for the individual brain lesions and their overlap. Columns are the observed brain areas, and rows their cytoarchitectonic probability classes. Colors indicate the relative over- (red) or under- (blue) representation of a structure-class in the lesion volume. White indicates no overlap between lesion and structure probability map, and black indicates probability classes that are not represented in the cytoarchitectonic map. $P_{\text {excess }}$ values indicate how much more likely a structure was observed in the lesion volume as could be expected from its own probability distribution, and thus reflect how central to the area the lesion volume is. ${ }^{41} \mathrm{BL}$, Basolateral; $\mathrm{CA}$, Cornu Ammonis, $\mathrm{CM}$, Central-Medial (which are all amygdala sub-regions); EC, Entorhinal Cortex; FD, Fascia Dentata (which are all bordering- or sub-regions of the hippocampus); HA, Hippocampal-Amygdaloid Transition Area; SC, Subicular Complex; SF, Superficial. 
a

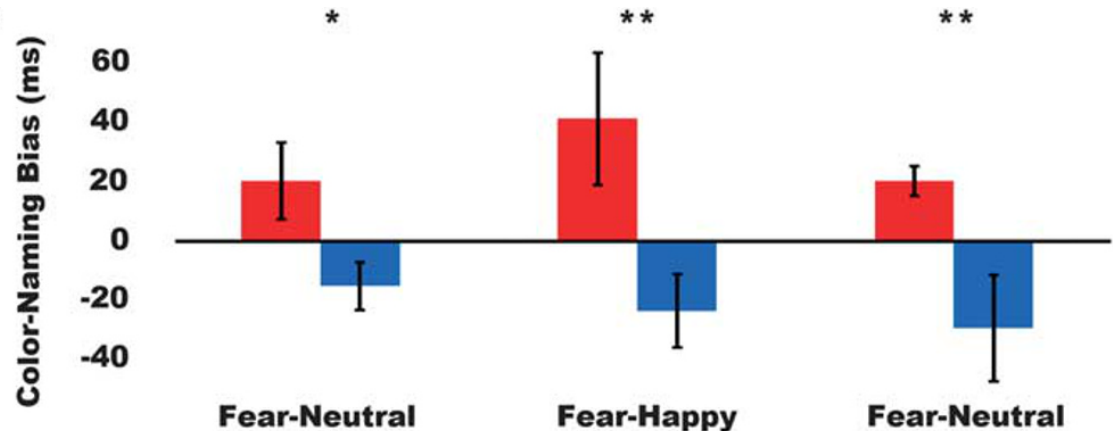

Fear-Neutral

Fear-Happy

$(n-1)$

b

Mean gaze-duration map
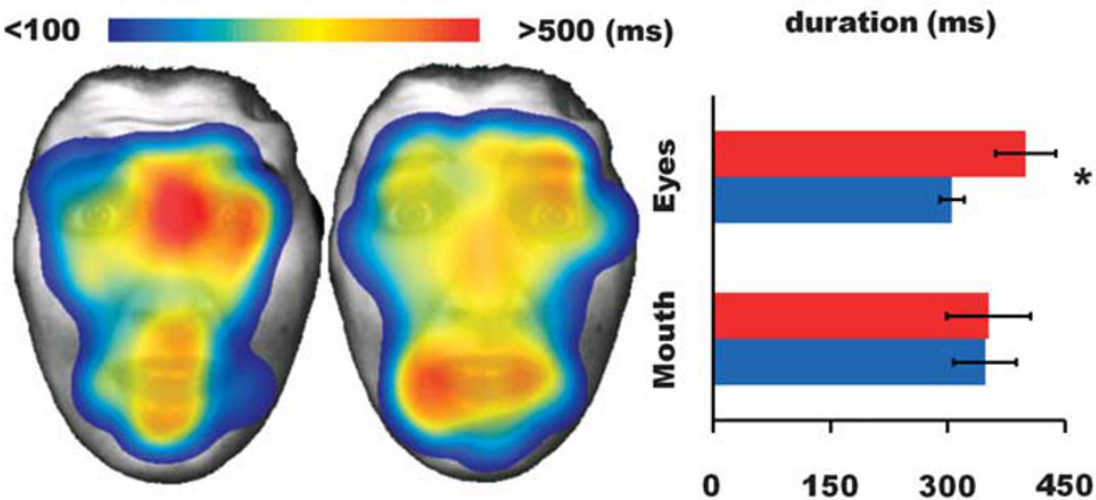

c

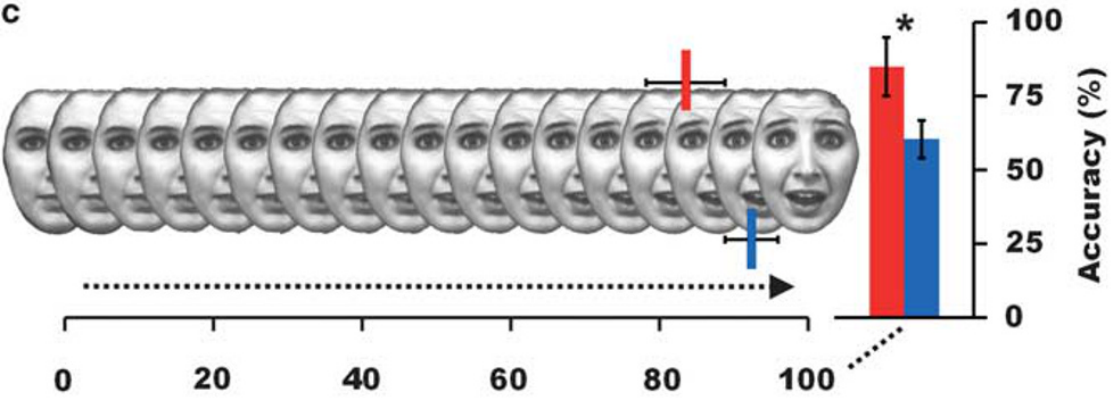

Fear sensitivity-point (morph-\%)

UWDs

Controls

Figure 4 Behavioral data. (a) Subliminal fear vigilance task. Bias scores were computed by subtracting mean latencies on neutral and happy trials from mean latencies on fear trials, and by subtracting mean latencies on neutral trials from fear trials that were preceded $(n-1)$ by neutral trials. Positive values represent slower color-naming responses when subliminally confronted with fearful faces compared with the control conditions, which is a reliable index of hypervigilance for subliminally presented threat cues. $^{35-37}$ (b) Eye-movement data from the dynamic emotion task. Mean duration of gaze fixations on the fear facial expressions; mapped on one of the stimuli, ${ }^{49}$ and quantified for the eye and mouth regions. (c) Performance data from the dynamic emotion task. Performance on fear recognition, sensitivity points for fear recognition indicating the average percentage of morphed fear necessary for consistent recognition, and recognition accuracy on the full-blown fear trials. See Supplementary Figure S2 for a detailed account on visual attention and performance on the static emotion task. Error bars represent s.e.m. ${ }^{*} P<0.05,{ }^{*} P<0.01$.

each emotion. As our hypothesis concerns fear processing in particular, we report the eye-movement data for the fear trials, and for all emotions pooled together.

UWD subjects and controls gazed overall equally long at the faces $(U=29, P=0.916)$, which indicates that reaction times for both groups were similar. UWD subjects directed $28 \%$ of their fixations to the mouth region, which was not significantly different $(U=24, P=0.527)$ from controls $(24 \%)$, and these fixations were similar in duration (376 ms vs $409 \mathrm{~ms}, U=25, P=0.598)$. Percentage of fixations directed to the eyes was also similar ( $17 \%$ vs $19 \%, U=26, P=0.673$ ), but these were significantly longer for UWD subjects $(381 \mathrm{~ms}$ vs $306 \mathrm{~ms}, U=11, P=0.045, r=0.49$ ). Thus, allocation of spatial attention was equal for both groups, but UWD subjects exhibited more sustained visual attention to dynamically presented eyes. 
Table 1 Emotion recognition data

\begin{tabular}{|c|c|c|c|c|c|c|}
\hline & \multicolumn{3}{|c|}{$\begin{array}{c}\text { Accuracy: } \\
\text { \%-correct (s.d.) }\end{array}$} & \multicolumn{3}{|c|}{$\begin{array}{c}\text { Sensitivity: } \\
\% \text {-morph (s.d.) }\end{array}$} \\
\hline & UWDs & Controls & $\mathbf{P}$ & UWDs & Controls & $\mathbf{P}$ \\
\hline Anger & & $92(12)$ & 0.150 & & & \\
\hline Disg & $45(27)$ & $60(38)$ & 0.3 & $4(23)$ & $83(20)$ & \\
\hline & $85(22)$ & 60 (23) & 0.0 & 2) & 92 (12) & \\
\hline piness & $100(0)$ & $100(0)$ & 1.00 & $28(15)$ & $26(12)$ & \\
\hline Sadness & $60(38)$ & $56(30)$ & 0.914 & $83(29)$ & $89(21)$ & 0.6 \\
\hline & $70(21)$ & & 0.7 & & 71 (28) & \\
\hline Tota & 71 (16) & 73 (13) & 0.830 & 72 (15) & 70 (10) & 0.9 \\
\hline
\end{tabular}

Abbreviation: UWD, Urbach-Wiethe disease.

Performance-scores (accuracy and sensitivity) with s.d. and $P$-values for the non-parametric Mann-Whitney $U$-tests for patients versus controls on the dynamic emotion recognition task.

Total time spent looking at dynamic fearful faces was equal for both groups $(U=27, P=0.752)$. UWD subjects directed $25 \%$ of all face fixations at the mouth region, which was not significantly different $(U=24, P=0.527)$ from controls $(20 \%)$. Number of fixations to the eye region of the fearful faces was also equal for both groups (20\% vs $23 \%, U=28, P=0.833$ ). Crucially, as in the whole-task analysis, duration of fixations at dynamically presented fearful eyes was longer for UWD subjects $(400 \mathrm{~ms}$ vs $305 \mathrm{~ms}, U=10, P=0.035, r=0.51$, see Figure $4 \mathrm{~b}$ ), while there was no duration difference for mouth fixations ( $353 \mathrm{~ms}$ vs $348 \mathrm{~ms}, U=27, P=0.752$, see Figure $4 \mathrm{~b}$ ). Thus, allocation of attention was similar for both groups, but UWD subjects exhibited prolonged attention to dynamically presented fearful eyes.

Sensitivity scores on the fear trials were not significantly different (84\% vs $92 \%, U=16.5, P=0.153)$, but in keeping with the hypothesis that visual attention to the eyes improves fear recognition ability, ${ }^{31}$ UWD subjects outperformed controls on full-blown fear trials ( $85 \%$ vs $60 \%$ correct, $U=12.5$, $P=0.048, r=0.48$, see Figure $4 c$ ).

Behavioral assessment: static emotion task. See Supplementary Information for a full account of these data. In short, performance was similar for UWD subjects and controls, but again UWD subjects showed near-significant longer fixation durations at the eyes of fearful faces $(U=12$, $P=0.058, r=0.46)$.

\section{Discussion}

Using a combination of structural and functional MRI as well as eye tracking and behavioral measures, we provide causal evidence that the human BLA acutely inhibits innate threat vigilance. Five UWD subjects with selective bilateral damage to the BLA show hypervigilance to subliminally presented fearful facial expressions. Moreover, they gazed longer at the eye region of, especially fearful, faces, while allocation of attention was similar to that of a carefully matched group of healthy controls. Following evidence that increased attention to the eye region of faces improves fear recognition performance, ${ }^{31}$ UWD subjects showed superior ability in dynamic fear recognition. These combined results establish that focal damage to the BLA makes humans hypervigilant to the innate cue for threat; fearful facial expressions.

To understand how BLA loss may lead to such hypervigilance, studies in animals provide valuable information. It is argued that the BLA inhibits the response to innate danger cues, because loss of BLA function in rodents leads to increased unconditioned fear. ${ }^{19-22} A$ possible neural pathway of such inhibition is through the CMA, ${ }^{23,55,56}$ which projects vastly to hypothalamic and brainstem areas, that regulate emotional responding through autonomic pathways. ${ }^{3}$ The CMA receives direct input from other parts of the amygdala, ${ }^{2,6,23}$ as well as from the PFC, ${ }^{5}$ and is considered to be the amygdala's behavioral output center, automatically allocating attention and directing autonomic and motor responses to threat. ${ }^{3,56-61}$ Importantly, the CMA is essential for the expression of active fear behaviors, as well as freezing responses generated in brainstem areas in response to acute threats. $^{62}$

The BLA is often regarded as the 'sensory' amygdala. It receives input from the sensory systems via the thalamus, ${ }^{60}$ as well as highly processed polymodal sensory information from association cortices including the PFC. ${ }^{2,5,40}$ The BLA is therefore argued to be involved in the automatic assessment of threat, ${ }^{16}$ and acquisition and extinction of conditioned fear. ${ }^{6,17,18}$ Furthermore, the BLA can through mutual connections with the PFC, especially the orbitofrontal cortex (OFC), both promote and inhibit the behavioral-output functions of the CMA, ${ }^{5,55,63-66}$ which might provide an explanation for the increased threat vigilance found in our UWD subjects.

An alternative explanation might be found in the recently discovered direct inhibitory functions of the BLA on the CMA in rodents. First, an inhibitory pathway from the lateral to medial $\mathrm{CMA}^{57,58}$ has been shown to switch CMA functions from the promotion of basal freezing responses to active threat assessment in the presence of an acute threat, ${ }^{56}$ and a direct projection from the BLA to this lateral CMA was recently reported to acutely reduce fearful behaviors. ${ }^{23}$ This landmark study showed that after optogenetic stimulation of BLA terminals in the lateral CMA fearful behavior decreased, and rodents started to explore potentially unsafe surroundings. Exploring was, however, significantly reduced when the same projection was inhibited. Importantly, no effect was observed after glutamergic stimulation of BLA somata, possibly reflecting the direct excitatory pathways from BLA to the medial CMA that can counteract the anxiolytic effects. It therefore seems that only acute stimulation of the BLA-CMA pathway reduces fearful behavior, ${ }^{23}$ which subsequently promotes the switch away from passive fear responding in the CMA. ${ }^{56}$ This notion is further supported by several studies showing that BLA deactivation increases unconditioned fear behavior and acute-freezing responses, while conditioned and more generalized fear is unaffected or even reduced. ${ }^{19-22}$

Furthermore, basal fear inhibition by the BLA could also be explained in terms of parallel models of amygdala functioning. In these models, the BLA, together with the NA (nucleus accumbens), is thought to be part of a system that underlies instrumental choice behaviors, whereas CMA-NA interactions sub-serve reflexive behavioral responding. ${ }^{67,68}$ Notably, for an efficient instrumental response to threats, the option for inhibitory control of reflexive fight-flight mechanisms is 
necessary, while in acutely threatening situations defensive reactivity can get priority. The latter is reflected in the switch from BLA-PFC to CMA-PAG activation when a threat becomes so proximal that it is unavoidable. ${ }^{25-27}$ Speculatively, the BLA might provide the necessary conditions for higher-order instrumental choice behaviors in mildly threatening situations. Following this model it might be expected that UWD subjects are more impulsive in decision making, but this needs to be confirmed by future research.

Note that the above-described mechanisms of acute fear regulation are independent from whether or not the amygdala has a direct role in the evaluation of threat-related information. Indeed, our UWD subjects are not impaired in emotion recognition, which suggests that the BLA does not contribute to conscious emotion recognition. Furthermore, responsivity to innate threat cues, like fearful faces, in sub-cortical areas is a survival reflex relatively independent from, but projecting to, the amygdala. ${ }^{16,69}$ Downregulation of such acute fear responsivity might therefore be the BLA's default mode, thereby reducing defensive reflexes and creating the conditions for a more instrumental response. Although conscious evaluation of emotional information thus seems not affected, it might be expected that reduced inhibition of such acute threat responding will affect our UWD subjects' ability to evaluate emotionally conflicting information correctly, but this remains to be tested.

In sum, the rodent BLA apparently can acutely inhibit fear responses to innate danger cues through its influence on the CMA, and our corresponding behavioral data in BLAdamaged subjects suggest that this BLA-CMA pathway may act in similar ways in humans. We do, however, have no insights into the intricate neural pathways with the present evidence, and the question whether the hypervigilance in our subjects with BLA damage is caused by loss of direct CMA modulation, indirectly via prefrontal areas, or both, awaits future research. Nonetheless, our data do show that damage to the BLA in humans leads to hypervigilant responses to innate threat cues. Such threat hypervigilance in humans is hypothetically related to acute fear and panic. ${ }^{4,24,25}$ Given the high prevalence of co-morbid anxiety disorders, including social phobia and panic disorder observed in UWD, ${ }^{34,70}$ our data provide important insights into the neural mechanisms of disorders of fear and anxiety.

Additionally, our UWD subjects showed superior performance on full-blown dynamic facial fear recognition. Such counterintuitive functional improvement associated with brain damage may reflect 'paradoxical functional facilitation', which refers to the fact that brain lesions sometimes can result in improved behavioral performance. ${ }^{71}$ This mechanism can be explained by considering the dynamic and active interplay of excitatory and inhibitory connections within neural circuits. When structure-A contributes to function- $X$, and structure- $B$ inhibits structure- $A$, loss of structure-B will relieve the inhibition of structure-A resulting in improvement of function$X$. Following our argument on reduced inhibition of fear vigilance in our UWD subjects, dynamic fearful faces could also evoke hypervigilance. It is indeed well established that passive viewing of fearful faces evokes simultaneous autonomic responses and amygdala activity. ${ }^{72-74}$ We therefore argue that failure of the BLA to inhibit these basal fear responses may engender upregulation of attentional vigilance mechanisms, and therefore hypervigilance to emotionally salient areas of faces, as seen in the increased fixation duration to, especially fearful, eyes. This increased processing of the eye region, might consequently result in the here observed paradoxical functional facilitation, ${ }^{71}$ in terms of improved fear recognition. ${ }^{31}$

Although future research should confirm whether fearful faces also evoke stronger autonomic responses in these subjects, the high prevalence of co-morbid anxiety disorders, including panic disorder observed in UWD, ${ }^{34,70}$ might be attributable to the same disinhibition phenomenon we propose, but in those cases causing secondary psychopathology. On the contrary, lack of, and hypo-attention for, fear, as seen in UWD-subject SM, ${ }^{31,75}$ might be due to the fact that her entire amygdala has been damaged. As has also been demonstrated in rodents, ${ }^{2}$ and primates, ${ }^{17,62,76}$ full amygdala damage can result in an inability to evaluate threats as salient, which might also explain why this UWD subject' is unable to automatically allocate attention to emotional salient information, ${ }^{31}$ while this function is fully intact in our UWD subjects.

\section{Conclusion}

Using a multimodal research strategy involving structural and functional MRI as well as eye-tracking and behavioral assessments, we show that five subjects with bilateral damage selective to the BLA, are hypervigilant for fearful facial expressions. Our lesion data can be uniquely translated to recent rodent studies, ${ }^{19-23,56}$ and provide the first direct evidence in support of the hypothesis that the human BLA inhibits acute hypervigilance to innate threat cues. These findings have important implications for the understanding of heterogeneous amygdala functions, and especially for the role of the BLA in the disorders of fear and anxiety.

\section{Conflict of interest}

The authors declare no conflict of interest.

Acknowledgements. The work in this paper was supported by grants from the Hope For Depression Research Foundation (HDRF: RGA no. 9-015), the Utrecht University (High-Potential program), the Netherlands Society of Scientific research (Brain and Cognition: no. 056-24-010), the South African MRC/DST Professional Development Program and the University of Cape Town (Brain Behavior Initiative).

1. Pessoa L, Adolphs R. Emotion processing and the amygdala: From a 'low road' to 'many roads' of evaluating biological significance. Nat Rev Neurosci 2010; 11: 773-783.

2. Davis M, Whalen PJ. The amygdala: vigilance and emotion. Mol Psychiatry 2001; 6: 13-34.

3. Heimer L, Harlan RE, Alheid GF, Garcia MM, de Olmos J. Substantia innominata: a notion which impedes clinical-anatomical correlations in neuropsychiatric disorders. Neuroscience 1997; 76: 957-1006.

4. McNaughton N, Corr PJ. A two-dimensional neuropsychology of defense: fear/anxiety and defensive distance. Neurosci Biobehav Rev 2004; 28: 285-305.

5. Barbas H, Saha S, Rempel-Clower N, Ghashghaei T. Serial pathways from primate prefrontal cortex to autonomic areas may influence emotional expression. BMC Neurosci 2003; $4: 25$.

6. Phelps EA, LeDoux JE. Contributions of the amygdala to emotion processing: from animal models to human behavior. Neuron 2005; 48: 175-187.

7. Hurlemann R, Wagner M, Hawellek B, Reich H, Pieperhoff P, Amunts K et al. Amygdala control of emotion-induced forgetting and remembering: evidence from Urbach-Wiethe disease. Neuropsychologia 2007; 45: 877-884. 
8. Hurlemann R, Schlaepfer TE, Matusch A, Reich H, Shah NJ, Zilles $\mathrm{K}$ et al. Reduced 5 $\mathrm{HT}(2 \mathrm{~A})$ receptor signaling following selective bilateral amygdala damage. Soc Cogn Affect Neurosci 2009; 4: 79-84.

9. Adolphs R, Tranel D, Damasio H, Damasio AR. Impaired recognition of emotion in facial expressions following bilateral damage to the human amygdala. Nature 1994; 372 669-672.

10. Adolphs R, Tranel D, Damasio H, Damasio AR. Fear and the human amygdala. J Neurosci 1995; 15: 5879-5891.

11. Adolphs R. Fear, faces, and the human amygdala. Curr Opin Neurobiol 2008; 18: 166-172.

12. Brand M, Grabenhorst F, Starcke K, Vandekerckhove MM, Markowitsch HJ. Role of the amygdala in decisions under ambiguity and decisions under risk: evidence from patients with Urbach-Wiethe disease. Neuropsychologia 2007; 45: 1305-1317.

13. Cahill L, Babinsky R, Markowitsch HJ, McGaugh JL. The amygdala and emotional memory. Nature 1995; 377: 295-296.

14. Hurlemann R, Patin A, Onur OA, Cohen MX, Baumgartner T, Metzler S et al. Oxytocin enhances amygdala-dependent, socially reinforced learning and emotional empathy in humans. J Neurosci 2010; 30: 4999-5007.

15. Siebert M, Markowitsch HJ, Bartel P. Amygdala, affect and cognition: evidence from 10 patients with Urbach-Wiethe disease. Brain 2003; 126: 2627-2637.

16. Liddell BJ, Brown KJ, Kemp AH, Barton MJ, Das $\mathrm{P}$, Peduto A et al. A direct brainstem-amygdala-cortical 'alarm' system for subliminal signals of fear. Neuroimage 2005; 24: 235-243.

17. Amaral DG. The amygdala, social behavior, and danger detection. Ann N Y Acad Sci 2003; 1000: 337-347.

18. Parkes SL, Westbrook RF. The basolateral amygdala is critical for the acquisition and extinction of associations between a neutral stimulus and a learned danger signal but not between two neutral stimuli. J Neurosci 2010; 30: 12608-12618.

19. Macedo CE, Cuadra G, Molina V, Brandao ML. Aversive stimulation of the inferior colliculus changes dopamine and serotonin extracellular levels in the frontal cortex: modulation by the basolateral nucleus of amygdala. Synapse 2005; 55: 58-66.

20. Macedo CE, Martinez RC, Brandao ML. Conditioned and unconditioned fear organized in the inferior colliculus are differentially sensitive to injections of muscimol into the basolateral nucleus of the amygdala. Behav Neurosci 2006; 120: 625-631.

21. Macedo CE, Martinez RC, Albrechet-Souza L, Molina VA, Brandao ML. 5-HT2- and D1mechanisms of the basolateral nucleus of the amygdala enhance conditioned fear and impair unconditioned fear. Behav Brain Res 2007; 177: 100-108.

22. Martinez RC, Ribeiro de Oliveira A, Brandao ML. Serotonergic mechanisms in the basolateral amygdala differentially regulate the conditioned and unconditioned fear organized in the periaqueductal gray. Eur Neuropsychopharmacol 2007; 17 717-724

23. Tye KM, Prakash R, Kim SY, Fenno LE, Grosenick L, Zarabi H et al. Amygdala circuitry mediating reversible and bidirectional control of anxiety. Nature 2011; 471: 358-362.

24. Graeff FG, Del-Ben CM. Neurobiology of panic disorder: from animal models to brain neuroimaging. Neurosci Biobehav Rev 2008; 32: 1326-1335

25. Maren S. The threatened brain. Science 2007; 317: 1043-1044.

26. Mobbs D, Petrovic P, Marchant JL, Hassabis D, Weiskopf N, Seymour B et al. When fear is near: threat imminence elicits prefrontal-periaqueductal gray shifts in humans. Science 2007; 317: 1079-1083

27. Mobbs D, Marchant JL, Hassabis D, Seymour B, Tan G, Gray M et al. From threat to fear: the neural organization of defensive fear systems in humans. J Neurosci 2009; 29: 12236-12243.

28. Costafreda SG, Brammer MJ, David AS, Fu CH. Predictors of amygdala activation during the processing of emotional stimuli: a meta-analysis of 385 PET and PMRI studies. Brain Res Rev 2008; 58: 57-70.

29. Etkin A, Klemenhagen KC, Dudman JT, Rogan MT, Hen R, Kandel ER et al. Individual differences in trait anxiety predict the response of the basolateral amygdala to unconsciously processed fearful faces. Neuron 2004; 44: 1043-1055.

30. Adolphs R. Investigating human emotion with lesions and intracranial recording. In: Allen J Coan J (eds). Handbook of Emotion Elicitation and Assessment. Oxford University Press: New York, 2007, pp 426-439.

31. Adolphs R, Gosselin F, Buchanan TW, Tranel D, Schyns P, Damasio AR. A mechanism for impaired fear recognition after amygdala damage. Nature 2005; 433: 68-72.

32. Gamer M, Buchel C. Amygdala activation predicts gaze toward fearful eyes. J Neurosci 2009; 29: 9123-9126.

33. Whalen PJ, Kagan J, Cook RG, Davis FC, Kim H, Polis S et al. Human amygdala responsivity to masked fearful eye whites. Science 2004; 306: 2061.

34. Thornton HB, Nel D, Thornton D, van Honk J, Baker GA, Stein DJ. The neuropsychiatry and neuropsychology of lipoid proteinosis. J Neuropsychiatry Clin Neurosci 2008; 20: $86-92$

35. Algom D, Chajut E, Lev S. A rational look at the emotional stroop phenomenon: a generic slowdown, not a Stroop effect. J Exp Psychol Gen 2004; 133: 323-338.

36. van Honk J, Schutter DJ, d'Alfonso AA, Kessels RP, de Haan EH. $1 \mathrm{hz}$ rTMS over the right prefrontal cortex reduces vigilant attention to unmasked but not to masked fearful faces. Biol Psychiatry 2002; 52: 312-317.

37. van Honk J, Peper JS, Schutter DJ. Testosterone reduces unconscious fear but not consciously experienced anxiety: implications for the disorders of fear and anxiety. Biol Psychiatry 2005; 58: 218-225.
38. Crinion J, Ashburner J, Leff A, Brett M, Price C, Friston K. Spatial normalization of lesioned brains: Performance evaluation and impact on fMRI analyses. Neuroimage 2007; 37: $866-875$.

39. Amunts K, Kedo O, Kindler M, Pieperhoff P, Mohlberg H, Shah NJ et al. Cytoarchitectonic mapping of the human amygdala, hippocampal region and entorhinal cortex: Intersubject variability and probability maps. Anat Embryol (Berl) 2005; 210: 343-352.

40. Solano-Castiella E, Anwander A, Lohmann G, Weiss M, Docherty C, Geyer S et al. Diffusion tensor imaging segments the human amygdala in vivo. Neuroimage 2010; 49: 2958-2965.

41. Eickhoff SB, Paus T, Caspers S, Grosbras MH, Evans AC, Zilles K et al. Assignment of functional activations to probabilistic cytoarchitectonic areas revisited. Neuroimage 2007; 36: $511-521$

42. Hariri AR, Mattay VS, Tessitore A, Kolachana B, Fera F, Goldman D et al. Serotonin transporter genetic variation and the response of the human amygdala. Science 2002; 297: 400-403.

43. Tottenham N, Tanaka JW, Leon AC, McCarry T, Nurse M, Hare TA et al. The NimStim set of facial expressions: Judgments from untrained research participants. Psychiatry Res 2009; 168: 242-249.

44. Friston KJ, Ashburner J, Frith CD, Poline J-B, Heather JD, Frackowiak RSJ. Spatial registration and normalization of images. Hum Brain Mapp 1995; 2: 165-189.

45. Eickhoff SB, Stephan KE, Mohlberg H, Grefkes C, Fink GR, Amunts $\mathrm{K}$ et al. A new SPM toolbox for combining probabilistic cytoarchitectonic maps and functional imaging data. Neuroimage 2005; 25: 1325-1335.

46. Ekman P, Friesen W. Pictures of Facial Affect. Consulting Psychologist Press: Palo Alto, 1976

47. Van Selst M, Merikle PM. Perception below the objective threshold? Conscious Cogn 1993; 2: 194-203.

48. Wells A, Matthews G. Attention and Emotion: a clinical perspective. Lawrence Erlbaum Associates, Inc.: Hillsdale, NJ, 1994

49. Lundqvist D, Flykt A, Ohman A. The Karolinska Directed Emotional Faces - KDEF CD ROM from Department of Clinical Neuroscience, Psychology Section. Karolinska Institutet, 1998

50. Tobii Technology. User Manual. Tobii Technology AB: Danderyd, Sweden, 2006.

51. Appenzeller S, Chaloult E, Velho P, de Souza EM, Araujo VZ, Cendes F et al. Amygdalae calcifications associated with disease duration in lipoid proteinosis. J Neuroimaging 2006; 16. 154-156.

52. Fusar-Poli P, Placentino A, Carletti F, Landi P, Allen P, Surguladze $S$ et al. Functional atlas of emotional faces processing: a voxel-based meta-analysis of 105 functional magnetic resonance imaging studies. J Psychiatry Neurosci 2009; 34: 418-432.

53. Etkin A, Egner T, Peraza DM, Kandel ER, Hirsch J. Resolving emotional conflict: a role for the rostral anterior cingulate cortex in modulating activity in the amygdala. Neuron 2006; 51: 871-882.

54. Kunde W, Mauer N. Sequential modulations of valence processing in the emotional stroop task. Exp Psychol 2008; 55: 151-156.

55. Barbas H. Specialized elements of orbitofrontal cortex in primates. Ann N Y Acad Sci2007; 1121: 10-32.

56. Gozzi A, Jain A, Giovanelli A, Bertollini C, Crestan V, Schwarz AJ et al. A neural switch for active and passive fear. Neuron 2010; 67: 656-666.

57. Ciocchi S, Herry C, Grenier F, Wolff SB, Letzkus JJ, Vlachos I et al. Encoding of conditioned fear in central amygdala inhibitory circuits. Nature 2010; 468: 277-282.

58. Haubensak W, Kunwar PS, Cai H, Ciocchi S, Wall NR, Ponnusamy R et al. Genetic dissection of an amygdala microcircuit that gates conditioned fear. Nature 2010; 468: 270-276.

59. Holland PC, Gallagher M. Amygdala circuitry in attentional and representational processes. Trends Cogn Sci 1999; 3: 65-73.

60. LeDoux JE. Emotional memory systems in the brain. Behav Brain Res 1993; 58: 69-79.

61. Mosher CP, Zimmerman PE, Gothard KM. Response characteristics of basolateral and centromedial neurons in the primate amygdala. J Neurosci 2010; 30: 16197-16207.

62. Kalin NH, Shelton SE, Davidson RJ. The role of the central nucleus of the amygdala in mediating fear and anxiety in the primate. J Neurosci 2004; 24: 5506-5515.

63. Garcia R, Vouimba RM, Baudry M, Thompson RF. The amygdala modulates prefrontal cortex activity relative to conditioned fear. Nature 1999; 402: 294-296.

64. Salzman CD, Fusi S. Emotion, cognition, and mental state representation in amygdala and prefrontal cortex. Annu Rev Neurosci 2010; 33: 173-202.

65. Quirk GJ, Likhtik E, Pelletier JG, Pare D. Stimulation of medial prefrontal cortex decreases the responsiveness of central amygdala output neurons. J Neurosci 2003; 23 : $8800-8807$.

66. Hampton AN, Adolphs R, Tyszka MJ, O'Doherty JP. Contributions of the amygdala to reward expectancy and choice signals in human prefrontal cortex. Neuron 2007; 55: 545-555.

67. Balleine BW, Killcross S. Parallel incentive processing: an integrated view of amygdala function. Trends Neurosci 2006; 29: 272-279.

68. Killcross S, Robbins TW, Everitt BJ. Different types of fear-conditioned behaviour mediated by separate nuclei within amygdala. Nature 1997; 388: 377-380.

69. LeDoux J. Rethinking the emotional brain. Neuron 2012; 73: 653-676.

70. Wiest G, Lehner-Baumgartner $\mathrm{E}$, Baumgartner $\mathrm{C}$. Panic attacks in an individual with bilateral selective lesions of the amygdala. Arch Neurol 2006; 63: 1798-1801. 
71. Kapur N. Paradoxical functional facilitation in brain-behaviour research. A critical review. Brain 1996; 119(Pt 5): 1775-1790.

72. Williams LM, Phillips ML, Brammer MJ, Skerrett D, Lagopoulos J, Rennie $C$ et al. Arousal dissociates amygdala and hippocampal fear responses: evidence from simultaneous fMR and skin conductance recording. Neuroimage 2001; 14: 1070-1079.

73. Williams LM, Barton MJ, Kemp AH, Liddell BJ, Peduto A, Gordon E et al. Distinct amygdala-autonomic arousal profiles in response to fear signals in healthy males and females. Neuroimage 2005; 28: 618-626.

74. Williams LM, Liddell BJ, Kemp AH, Bryant RA, Meares RA, Peduto AS et al. Amygdalaprefrontal dissociation of subliminal and supraliminal fear. Hum Brain Mapp 2006; 27 652-661.

75. Feinstein JS, Adolphs R, Damasio A, Tranel D. The human amygdala and the induction and experience of fear. Curr Biol 2011; 21: 34-38.
76. Kalin NH, Shelton SE, Engeland CG, Haraldsson HM, Marucha PT. Stress decreases, while central nucleus amygdala lesions increase, IL-8 and MIP-1alpha gene expression during tissue healing in non-human primates. Brain Behav Immun 2006; 20 564-568.

\section{(c)}

Translational Psychiatry is an open-access journal published by Nature Publishing Group. This work is licensed under the Creative Commons Attribution-Noncommercial-No Derivative Works 3.0 Unported License. To view a copy of this license, visit http://creativecommons.org/licenses/by-nc-nd/3.0/

Supplementary Information accompanies the paper on the Translational Psychiatry website (http://www.nature.com/tp) 